\title{
The role of FOLFIRINOX in metastatic pancreatic cancer: a meta-analysis
}

\author{
Beilei Zhang ${ }^{1}$, Fengyan Zhou ${ }^{2}$, Jiaze Hong ${ }^{1}$, Derry Minyao $\mathrm{Ng}^{3}$, Tong Yang ${ }^{4}$, Xinyu Zhou', Jieyin Jin ${ }^{1}$, Feifei Zhou',
} Ping Chen ${ }^{5}$ and Yunbao $\mathrm{Xu}^{6^{*}}$

\begin{abstract}
Background: The prognosis of pancreatic cancer (PC) is extremely poor, and most patients with metastatic PC still receive palliative care. Here, we report the efficacy and safety of FOLFIRINOX (oxaliplatin, irinotecan, leucovorin, 5fluorouracil) in the treatment of metastatic PC.

Methods: We searched PubMed, Web of Science, EBSCO, and Cochrane library databases for articles that described efficacy and safety of FOLFIRINOX in patients with metastatic PC, from January 1996 to July 2020. The primary outcomes targeted included overall survival (OS) and progression-free survival (PFS).

Results: We found that FOLFIRINOX could directly improve OS rate of patients with metastatic PC (HR $0.76,95 \% \mathrm{Cl}$ $0.67-0.86, p<0.001)$ but had no benefit on PFS. Results from subgroup analyses showed that FOLFIRINOX had superior benefits than monochemotherapy (HR 0.59, 95\% Cl 0.52-0.67, $p<0.001$ ), followed by FOLFIRINOX versus combination chemotherapy (HR 0.76, 95\% Cl 0.61-0.95, $p<0.001$ ). The result of FOLFIRINOX versus nab-paclitaxel + gemcitabine had no benefit (HR 0.91, 95\% Cl 0.82-1.02, p>0.05). The main adverse events (AEs) targeted hematological toxicity and the gastrointestinal system, and included febrile neutropenia, a reduction in white blood cells and appetite, as well as diarrhea.

Conclusion: These findings indicated that FOLFIRINOX has potential benefits for the prognosis of patients with metastatic PC. Furthermore, there is no difference between the regimen of FOLFIRINOX and nab-paclitaxel + gemcitabine in this study. The application of FOLFIRINOX should be according to the actual situation of the patients and the experience of the doctors.
\end{abstract}

Keywords: Metastatic pancreatic cancer, FOLFIRINOX, Efficacy, Safety, Meta-analysis

\section{Introduction}

The 2018 Global Cancer Incidence Research Report, based on 185 countries, regards pancreatic cancer (PC) as the seventh leading cause of cancer-related deaths worldwide [1]. PC has an extremely poor prognosis, with a 5 -year relative survival rate of only $8 \%$ [2]. This is attributed to the fact that PC cases are either locally invasive or metastasized at diagnosis [3]. Despite recent

\footnotetext{
* Correspondence: chenjj1984no2h@163.com

${ }^{6}$ Department of Radiotherapy and Chemotherapy, Hwamei Hospital, University of Chinese Academy of Sciences, Northwest Street 41, Haishu District, Ningbo 315010, Zhejiang, China

Full list of author information is available at the end of the article
}

progress in developing treatment therapies that improve PC outcomes, to date, approximately $20 \%$ of all patients survive at 1 year [4]. In fact, most approaches for treating metastatic PC still use palliative therapy [5]. A randomized trial by Burris et al. showed that gemcitabine resulted in a moderate survival advantage, compared to 5-FU treatment, as evidenced by median survival times of 5.65 and 4.41 months, respectively $(p=0.0025)$ [6]. Consequently, gemcitabine has become a reference plan and the first-line drug for clinical treatment of metastatic PC [7]. On the other hand, previous studies have demonstrated the anti-tumor activity of irinotecan and

(c) The Author(s). 2021 Open Access This article is licensed under a Creative Commons Attribution 4.0 International License, which permits use, sharing, adaptation, distribution and reproduction in any medium or format, as long as you give appropriate credit to the original author(s) and the source, provide a link to the Creative Commons licence, and indicate if changes were made. The images or other third party material in this article are included in the article's Creative Commons licence, unless indicated otherwise in a credit line to the material. If material is not included in the article's Creative Commons licence and your intended use is not permitted by statutory regulation or exceeds the permitted use, you will need to obtain permission directly from the copyright holder. To view a copy of this licence, visit http://creativecommons.org/licenses/by/4.0/. The Creative Commons Public Domain Dedication waiver (http://creativecommons.org/publicdomain/zero/1.0/) applies to the data made available in this article, unless otherwise stated in a credit line to the data. 
oxaliplatin against several solid tumors, especially in gastrointestinal tumor cell lines [8]. In addition, a combination of leucovorin and 5-FU-based therapies applied for treatment of metastatic colorectal cancer showed improved efficacy and toxicity [9]. Conroy et al. conducted a randomized trial to explore the effectiveness of FOLFIRINOX (oxaliplatin, irinotecan, leucovorin, 5fluorouracil) versus gemcitabine as a first-line chemotherapy regimen in patients with metastatic PC, and found that FOLFIRINOX exerted significant survival benefits $(\mathrm{HR}, 0.54 ; p<0.001)$ relative to gemcitabine, suggesting its potential as a first-line treatment for patients with metastatic PC [10]. We hypothesized that FOLFIRINOX may have toxicity-related problems, since it was combined with other chemotherapy drugs. Therefore, we conducted a meta-analysis on the efficacy and toxicity of FOLFIRINOX as a chemotherapy regimen, relative to other chemotherapies in patients with metastatic PC. Our findings are expected to reveal its benefits in patients with metastatic PC.

\section{Methods}

\section{Literature search strategy}

This meta-analysis was conducted according to the Preferred Reporting Item of the Systematic Review and Meta-Analysis Agreement (PRISMA-P) 2015 [11]. To identify relevant research articles describing the effect of FOLFIRINOX in unresectable PC, we systematically searched various electronic databases, including PubMed, Web of Science, EBSCO, and Cochrane Library, from January 1996 to July 2020. We used the following search terms to filter related articles: "FOLFOXIRI", "mFOLFOXIRI", "modified FOLFOXIRI", "FOLFIRINOX", "mFOLFIRINOX", "modified FOLFIRINOX", "irinotecan", "oxaliplatin", "leucovorin", "5-fluorouracil", "pancreatic cancer", "pancreatic carcinoma", "carcinoma of the pancreas", "cancer of the pancreas". Two researchers independently reviewed the articles' abstracts, according to our selection criteria, then examined review articles and references of all retrieved articles to obtain other potentially relevant items. There is no additional registration information for this study.

\section{Inclusion and exclusion criteria}

Articles that met the following criteria were included in the analysis: (1) studies were randomized controlled trials (RCTs) or observational studies; (2) all patients were diagnosed with unresectable PC; (3) patients in the experimental group received FOLFIRINOX or modified FOLFIRINOX regimens, whereas those in the control group received only monotherapy or other chemotherapy regimens; (4) survival outcomes, including OS and PFS, were extractable; and (5) in case of duplicate or constantly updated publications, the latest article was used in this study. Conversely, articles that met the following criteria were excluded: (1) those reporting nonoriginal research; (2) research population comprising non-metastatic PC patients, such as postoperative adjuvant or neoadjuvant chemotherapy; (3) studies that did not compare FOLFIRINOX with other chemotherapy; (4) appropriate data could not be obtained; and (5) articles not written in English.

\section{Outcome measures}

Retrieved articles reported different survival outcomes, including OS and PFS. Briefly, OS was defined as the length of time at the start of treatment to the date of death from any cause, whereas PFS referred to the length of time between initiation of therapy and objective tumor progression or death [12]. Hazard ratios (HR)/ relative ratios (RR), including 95\% confidence interval (CI), were used to assess the effect of FOLFIRINOX in metastatic PC. Data were directly extracted from the article or estimated according to the Kaplan-Meier survival curve.

\section{Data extraction and assessment of the risk of bias}

Data from each study were independently extracted by two researchers, using a pre-designed data extraction table. In case of disagreements, a third researcher was invited to reach a majority opinion [13]. The recorded information included baseline characteristics such as author, year of publication, tumor type, study type, therapeutic regimen, and sample size, as well as survival outcomes including OS, PFS, and adverse events (AEs). We assessed the risks of bias in the RCTs using the Cochrane Collaboration Risk of Bias Assessment Tool, then evaluated methodologic quality of observational studies using the Newcastle-Ottawa Quality Assessment Scale [14].

\section{Statistical analysis}

All data analyses were performed in the Stata 12.0 software (Stata, College Station). Briefly, heterogeneity among studies was evaluated using the Cochran chisquare test [15] and heterogeneity statistic $\left(\mathrm{I}^{2}\right)$ [16], whereas HR estimates were weighted and pooled using the Mantel-Haenszel random effects model to increase credibility by the regimen and the diversity of the population. In addition, we evaluated publication bias using Egger's test $[17,18]$ and performed sensitivity analysis to assess stability of the results. Data followed by $p<0.05$ were considered statistically significant.

\section{Results}

Eligible research and inclusion characteristics

Our search strategy resulted in a total of 1907 studies on FOLFIRINOX. After excluding non-metastatic PC, non- 
FOLFIRINOX compared with other chemotherapy, nonoriginal, as well as studies whose data could not be extracted, a total of 18 articles [5, 10, 19-34] were finally included in our analysis. A detailed outline of the search and selection process is shown in Fig. 1. The included articles comprised RCT and retrospective studies, respectively, one and seventeen $[5,10,19-34]$.

Eight studies [5, 10, 20, 23, 26, 29, 31, 33] described use of FOLFIRINOX versus monochemotherapy; most of which were FOLFIRINOX versus gemcitabine alone, 15 articles [5, 20-25, 27-34] related to FOLFIRINOX versus nab-paclitaxel combined with gemcitabine, whereas the rest reported FOLFIRINOX versus other chemotherapy regimens. The studies analyzed a total of 7556 participants, 2435 of whom received FOLFIRINOX. Among them, Kordes et al.'s research experimental group was FOLFOXIRI (oxaliplatin, irinotecan, 5fluorouracil) [23]. Detailed characteristics of the patients are shown in Supplementary Table 1. We also assessed the quality of all incorporated retrospective as well as RCT studies using the Newcastle-Ottawa Quality
Assessment Scale and the Cochrane Collaboration's tool, respectively. Both sets of results showed reliable article quality (Supplementary Table 2).

\section{Effect of FOLFIRINOX on overall survival}

In the study cohort, 17 studies [5, 10, 19-24, 26-34] reported OS with a combined total effect rate (HR 0.76, 95\% Cl 0.67-0.86, $p<0.001$; Fig. 2). Due to the considerable heterogeneity of the results, we performed a subgroup analysis on OS. Although we conducted a subgroup analysis, the high heterogeneity was still inevitable. This was due to the diversity of the population and regimen we included, and it is precisely because of this that we used the random effects model in the selection of the model. It found that FOLFIRINOX had superior benefits compared with monochemotherapy, with a combined total effective rate of $\mathrm{HR} 0.59,95 \% \mathrm{Cl} 0.52-$ 0.67, $p<0.001$ (Supplementary Fig. 1). This was followed by FOLFIRINOX versus combination chemotherapy, with a combined total effective rate of $\mathrm{HR} 0.76,95 \% \mathrm{Cl}$ $0.61-0.95, p<0.001$ (Supplementary Fig. 2). The result of

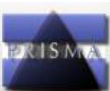

PRISMA 2009 Flow Diagram
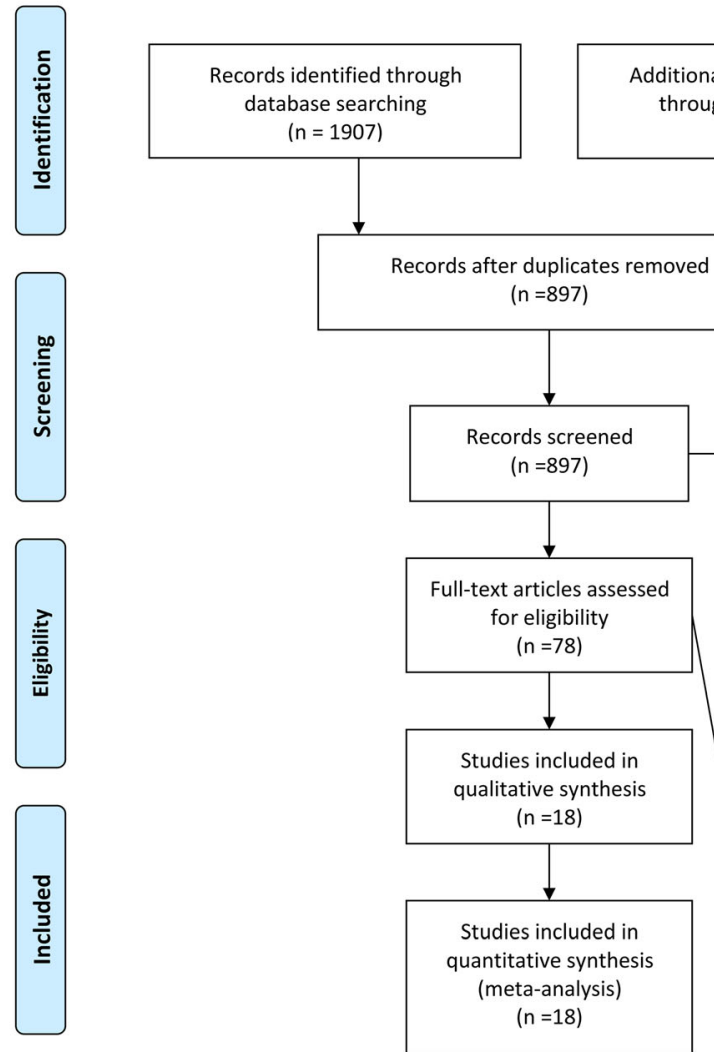

records identified rough other sources $(n=0)$ 


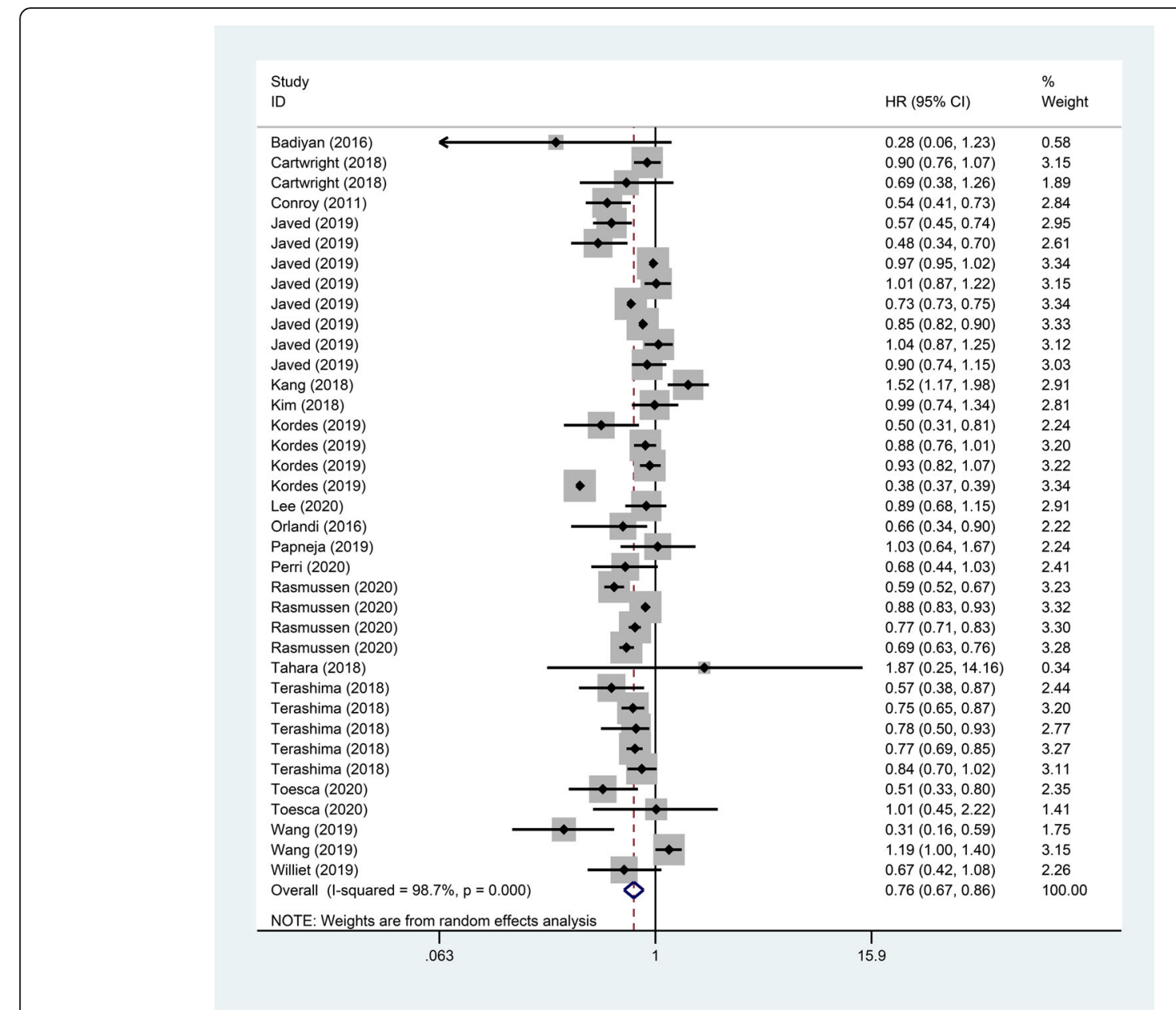

Fig. 2 Forest plots of the overall survival for FOLFIRINOX on metastatic pancreatic cancer

FOLFIRINOX versus nab-paclitaxel + gemcitabine had no benefit (HR 0.91, 95\% Cl 0.82-1.02, $p>0.05$; Fig. 3).

\section{Effect of FOLFIRINOX on progression-free survival}

We found eight studies [10, 21, 24-26, 32-34] that used PFS as endpoints, and subsequently combined them using statistical methods. The results revealed no benefit to PFS in the experimental group using FOLFIRINOX, relative to the control group (HR $0.88,95 \% \mathrm{Cl} 0.63-$ 1.22, $p>0.05$; Supplementary Fig. 3).

\section{AEs of FOLFIRINOX}

Among the included articles, a total of 12 studies [5, 10, 21-25, 27-31] described AEs. Analysis showed that total AEs were all hematological toxicity and gastrointestinal events, and included febrile neutropenia $(R R=2.19)$, decreased white blood cell $(R R=1.54)$, and low appetite $(R R=1.58)$, as well as diarrhea ( $R R=2.72)$, mucositis $(R R=$ $3.84)$, nausea $(R R=1.94)$, and vomiting $(R R=1.63)$. Significant AEs, among those rated grade 3 and higher, included febrile neutropenia $(R R=2.84)$, neutropenia $(R R=$
1.67), white blood cell decreased $(R R=3.09)$, diarrhea $(R R=3.74)$, elevated ALT $(R R=0.38)$, nausea $(R R=3.60)$, and vomiting $(\mathrm{RR}=1.46)$ (Table 1$)$.

\section{Publication bias and sensitivity of the OS of FOLFIRINOX}

Due to the relatively large number of articles included in our analysis, we evaluated publication bias to ascertain the relationship between OS and FOLFIRINOX. Egger $p=0.446$, showing there was no publication bias. Results from the sensitivity analysis showed stability.

\section{Discussion}

Our results showed that FOLFIRINOX was the most potentially beneficial regimen in metastatic PC, but in terms of long-time benefits, there was no statistical difference compared with nab-paclitaxel + gemcitabine. Interestingly, Kang et al. [21] and Papneja et al. [27] also reported that FOLFIRINOX had no significant benefit versus nab-paclitaxel + gemcitabine in patients with metastatic PC. However, Suker et al. [35] found that FOLFIRINOX could prolong the overall survival of 


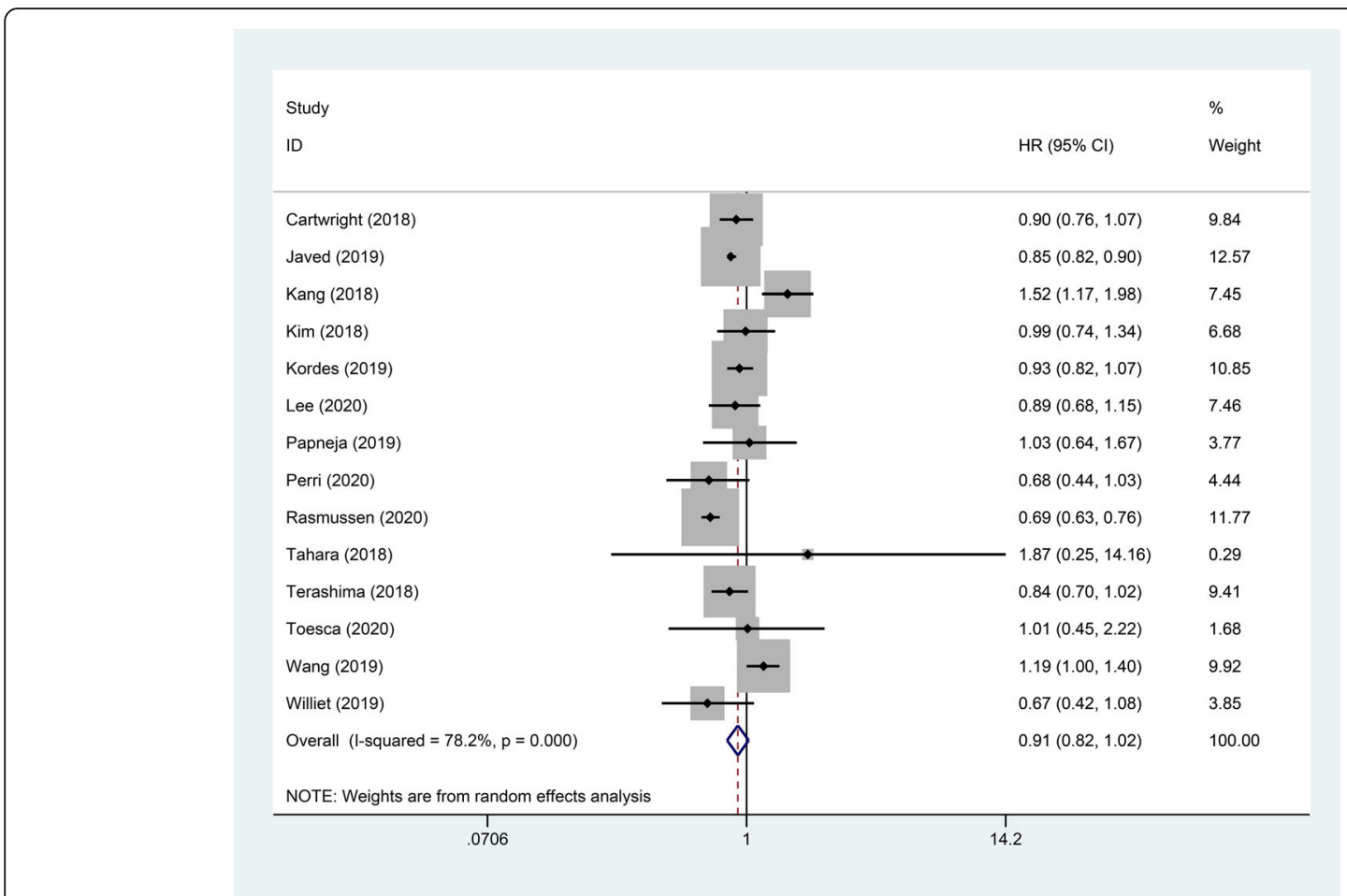

Fig. 3 Forest plot of the overall survival for FOLFIRINOX versus nab-paclitaxel+gemcitabine on metastatic pancreatic cancer

patients with locally advanced PC by comparison of FOLFIRINOX and gemcitabine. Interestingly, two studies of neoadjuvant chemotherapy based on FOLFIRINOX have also been confirmed to benefit patients with locally advanced PC [36, 37]. Furthermore, FOLFIRINOX and nab-paclitaxel + gemcitabine had superior benefits to the other group with regards to survival of patients with metastatic $\mathrm{PC}$, consistent with other recent studies that reported similar findings [20, 23, 29].

From an OS perspective, the use of total FOLFIRINOX can benefit patients with metastatic PC, owing to a direct increase in the OS rate of disease control. However, results from our heterogeneity analysis were relatively large, necessitating further validation. In addition, results from subgroup analysis of the heterogeneity indicated that FOLFIRINOX exerted survival benefits irrespective of whether the control group comprised monotherapy or gemcitabine-based combination chemotherapy, consistent with Orlandi et al. [26] and Terashima et al. [31] who found significantly higher survival benefits in the FOLFIRINOX relative to the control group. Similarly, results from a retrospective study on unresectable PC [32] also showed that FOLFIRINOX may have a survival benefit. Conversely, reported contrasting results [30]. We hypothesized that the conclusion of Tahara et al. might be attributed to the small sample size used in their study. Because the control group of this article contained different chemotherapy regimens, from the perspective of the first-line chemotherapy regimen of nab-paclitaxel + gemcitabine as the control group, our results proved that the regimen of FOLFIRINOX has potential survival benefit trend on patients with metastatic PC, consistent with previous studies $[5,28]$.

FOLFIRINOX had superior OS benefits relative to monotherapy, followed by FOLFIRINOX versus gemcitabine-based combination chemotherapy not included nab-paclitaxel + gemcitabine. In the monotherapy and combination chemotherapy regimen, gemcitabine accounted for the majority of the benefit. Therefore, we attributed this difference in efficacy to the mechanism of drug action. As a deoxycytidine analog of gemcitabine, its cytotoxic activity was based on several activities of DNA synthesis. Functionally, structural differences between the fluorine substituents on the 2'position of the furanose ring of gemcitabine gives gemcitabine the unique cellular pharmacological characteristics, metabolism, and mechanism action with other nucleoside analogs [38]. However, gemcitabine is highly resistant, which appears within a few weeks of chemotherapy [39]. The mechanism may be caused by an alteration of gemcitabine drug metabolism that causes incorporation of cytidine analogs into DNA [40], or it may be related to a reduction of gemcitabine-induced apoptosis [41, 42].

Results from our subgroup analysis of FOLFIRINOX versus nab-paclitaxel + gemcitabine showed that the 
Table 1 Subgroup analysis of the adverse events (AEs)

\begin{tabular}{|c|c|c|c|c|c|}
\hline FOLFIRINOX vs. control & No. of studies & RR & $95 \% \mathrm{Cl}$ & $p$ & Heterogeneity $\left(I^{2}\right)$ \\
\hline Any grade about neuropathy & 6 & 1.12 & $0.60-2.10$ & 0.72 & 83 \\
\hline Any grade anemia & 8 & 0.95 & $0.59-1.52$ & 0.84 & 94 \\
\hline Any grade decreased appetite & 3 & 1.58 & $1.08-2.30$ & 0.02 & 78 \\
\hline Any grade diarrhea & 6 & 2.72 & $1.60-4.61$ & $<0.001$ & 74 \\
\hline Any grade fatigue & 5 & 1.25 & $0.92-1.71$ & 0.16 & 70 \\
\hline Any grade febrile neutropenia & 4 & 2.19 & $1.04-4.65$ & 0.04 & 25 \\
\hline Any grade mucositis & 2 & 3.84 & $2.33-6.34$ & $<0.001$ & 0 \\
\hline Any grade nausea & 4 & 1.94 & $1.16-3.24$ & 0.01 & 46 \\
\hline Any grade neutropenia & 8 & 1.15 & $0.86-1.52$ & 0.35 & 90 \\
\hline Any grade thrombocytopenia & 7 & 0.76 & $0.42-1.36$ & 0.36 & 92 \\
\hline Any grade vomiting & 4 & 1.63 & $1.26-2.09$ & $<0.001$ & 0 \\
\hline Any grade white blood cell decrease & 2 & 1.54 & $1.16-2.04$ & 0.003 & 6 \\
\hline Grade 3 or higher about neuropathy & 7 & 0.85 & $0.33-2.18$ & 0.73 & 62 \\
\hline Grade 3 or higher anemia & 9 & 1.21 & $0.73-2.03$ & 0.46 & 31 \\
\hline Grade 3 or higher decreased appetite & 2 & 1.41 & $0.56-3.57$ & 0.47 & 0 \\
\hline Grade 3 or higher diarrhea & 8 & 3.74 & $1.62-8.62$ & 0.002 & 54 \\
\hline Grade 3 or higher elevated ALT & 3 & 0.38 & $0.21-0.68$ & 0.001 & 0 \\
\hline Grade 3 or higher fatigue & 6 & 0.95 & $0.62-1.45$ & 0.80 & 35 \\
\hline Grade 3 or higher febrile neutropenia & 6 & 2.84 & $1.35-5.99$ & 0.006 & 30 \\
\hline Grade 3 or higher mucositis & 2 & 1.34 & $0.71-2.51$ & 0.37 & NA \\
\hline Grade 3 or higher nausea & 4 & 3.60 & $1.26-10.30$ & 0.02 & 11 \\
\hline Grade 3 or higher neutropenia & 8 & 1.67 & $1.14-2.46$ & 0.009 & 87 \\
\hline Grade 3 or higher thrombocytopenia & 9 & 1.20 & $0.74-1.93$ & 0.47 & 18 \\
\hline Grade 3 or higher vomiting & 6 & 1.46 & $1.07-1.98$ & 0.02 & 0 \\
\hline Grade 3 or higher white blood cell decrease & 2 & 3.09 & $2.00-4.77$ & $<0.001$ & 0 \\
\hline Death & 2 & 0.49 & $0.20-1.23$ & 0.13 & 0 \\
\hline
\end{tabular}

$R R$ risk ratio, $A L T$ alanine aminotransferase, $N A$ not applicable

Data in italics are statistically siginificant $(P<0.05)$

overall trend was biased towards the regimen of FOLFIRINOX; however, there was no statistical difference, which may be related to the small sample size. With the expansion of the sample size, the benefit trend of FOLFIRINOX may appear. For the combination chemotherapy of nab-paclitaxel + gemcitabine, its benefits were crucial in clinical treatment. Related studies have shown that the combination of nab-paclitaxel + gemcitabine can increase the drug concentration of gemcitabine in tumor cells, thereby causing cytotoxicity [43]. And this combination medication program was widely used in clinical practice. For the regimen of FOLFIRINOX, given the relatively large toxicity of FOLFIRINOX reported in previous studies and the higher physical requirements of this program [44], the population's clinical application is not extensive and needs careful consideration. Furthermore, PFS had no benefit, possibly due to the small sample size and insufficient follow-up time [25, 26]. This may also be attributed to the difference between indicators setting up in each article and the type of study (whether it is RCT). Despite FOLFIRINOX's superior benefits compared to the control regimen, further research is needed to evaluate its clinical value.

In the FOLFIRINOX program, previous studies have shown that irinotecan exerts specific clinical activity in patients with metastatic PC $[45,46]$. Similarly, preclinical studies by Azrak et al. [47], Mans et al. [48], and Mullany et al. [49] have shown that a combination of irinotecan with calcium folinate and fluorouracil produces significant synergistic effect [50]. Interestingly, clinical trials by Ducreux et al. [51] revealed that oxaliplatin produces individual clinical activity against PC only when combined with fluorouracil. Furthermore, a synergistic effect is produced when combined with irinotecan in vitro [52]. Consequently, Ychou et al. [8] and Conroy et al. [53] explored the benefits of irinotecan, oxaliplatin, fluorouracil, and calcium leucovorin in patients with 
metastatic PC and found that FOLFIRINOX had encouraging clinical benefits.

Researchers need to consider both the efficacy and toxicity before extensive clinical applications. Therefore, anticancer drugs' AEs are a vital consideration in clinical oncology [54]. AEs associated with FOLFIRINOX mainly targeted the blood and gastrointestinal systems. We speculated that this might be positively correlated with the mechanism of action of the drug itself. Previous studies have shown that, as a third-generation platinum compound [55], bone marrow suppression is a joint $\mathrm{AE}$ of oxaliplatin, and can cause blood system toxicity such as thrombocytopenia. Other studies have shown that the blood system toxicity of oxaliplatin may also be related to the immune-dependent mechanism [56] and the induced spleen enlargement [57]. Regarding the gastrointestinal system, we speculated that $\mathrm{AE}$ occurrence might be related to the anatomical location of the tumor.

FOLFIRINOX toxicity is a problem that cannot be ignored. This is because it had clear benefits, but was also accompanied by relatively large toxicities [58]. Consequently, mFOLFIRINOX has been employed as a potential agent for reducing toxicity and achieved significant curative effects. For instance, Kang et al. [59] and Ghorani et al. [60] reported that mFOLFIRINOX generated comparable efficacies to FOLFIRINOX in patients with metastatic PC, and this was accompanied by weak toxicity. They suggested that if clinically necessary, $75 \%$ of the standard dose should be used for treatment, and can alleviate the toxicity without affecting the efficacy. Unfortunately, this study did not include more related clinical trials on mFOLFIRINOX. Therefore, regarding the problem of FOLFIRINOX toxicity, we have put forward the following suggestions: (1) in the choice of clinical regimen, we recommend using $75 \%$ of the standard dose to treat patients with metastatic PC; (2) we recommend regular monitoring of the patient's various indicators to understand the patient's physical function during the treatment process; (3) in the selection of patients with metastatic PC, we recommend more patients with higher physical fitness scores to receive the treatment of this program in order to achieve better curative effects [61]. All in all, there are more clinical trials needed to evaluate ways of minimizing FOLFIRINOX toxicity to enhance its use in the clinical treatment of metastatic PC.

\section{Limitations}

Our analysis included several retrospective studies, which could have compromised accuracy of our results. Even though a few non-metastatic PC cases are contained in the included 12 articles, we have no way to obtain data for subgroup analysis. Thus, the subject of our study is metastatic PC, and we are discussing according to the treatments. In addition, the diverse treatment options in the control group may have generated potential heterogeneity, although we comprehensively and systematically compared efficacy of FOLFIRINOX as the experimental group in patients with metastatic PC. Nevertheless, the different medication regimens used indicated that FOLFIRINOX had more apparent benefits affirming our conclusions. However, these conclusions need to be validated using a large number of clinical trials.

\section{Strengths}

Although this article has the above limitations, it can still provide further strong evidence for the chemotherapy of metastatic PC, and it is hopeful to be widely used in clinical practice for the treatment of metastatic PC.

\section{Conclusion}

FOLFIRINOX is the potentially optimal regimen for the prognosis of patients with metastatic PC. Although the AEs increased at different degrees, FOLFIRINOX is generally safe and tolerable. There is no difference between the regimen of FOLFIRINOX and nab-paclitaxel + gemcitabine in this study. Therefore, it remains debatable whether the regimen of FOLFIRINOX can replace the combination chemotherapy of nab-paclitaxel + gemcitabine and the selection of the specific implementation plan should be according to the actual situation of the patients and the experience of the doctors.

\section{Abbreviations}

PC: Pancreatic cancer; FOLFIRINOX: Oxaliplatin, irinotecan, leucovorin, 5fluorouracil; FOLFOXIRI: Oxaliplatin, irinotecan, 5-fluorouracil; OS: Overall survival; PFS: Progression-free survival; AEs: Adverse events; HR: Hazard ratios; RR: Relative risk; Cl: Confidence interval; RCT: Randomized controlled trial

\section{Supplementary Information}

The online version contains supplementary material available at https://doi. org/10.1186/s12957-021-02291-6.

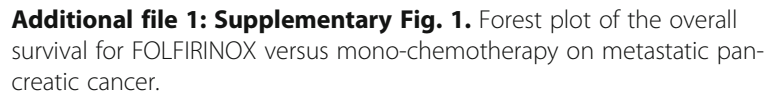

Additional file 2: Supplementary Fig. 2. Forest plot of the overall survival for FOLFIRINOX versus combination chemotherapy on metastatic pancreatic cancer.

Additional file 3: Supplementary Fig. 3. Forest plots of the progression-free survival for FOLFIRINOX on metastatic pancreatic cancer. Additional file 4: Supplementary Table 1. Characteristics of included clinical trials in the meta-analysis.

Additional file 5: Supplementary Table 2. Quality assessment of studies included.

\section{Acknowledgements}

None.

Authors' contributions

Each author contributed significantly to concept and development of the present paper. YBX and NND designed the research process. FYZ and JZH 
searched the database for corresponding articles. DMN and TY extracted useful information from the articles above. XYZ and JYJ used statistical software for analysis. YBX and NND drafted the meta-analysis. FFZ and PC polished this article. The authors read and approved the manuscript and ensured that this was the case.

\section{Funding}

The authors have no financial support to declare.

\section{Availability of data and materials}

The datasets supporting the conclusions of this article are included within the article.

\section{Declarations}

\section{Ethics approval and consent to participate}

This study did not require ethical approval since it was a review of published articles and did not directly involve the use of human or animal subjects.

\section{Consent for publication}

Not applicable.

\section{Competing interests}

The authors declare that they have no competing of interests.

\begin{abstract}
Author details
${ }^{1}$ The Second Clinical Medical College, Zhejiang Chinese Medical University, Hangzhou, Zhejiang, China. ${ }^{2}$ Emergency Medical Center, Ningbo Yinzhou No 2 Hospital, Ningbo, Zhejiang, China. ${ }^{3}$ Medical College of Ningbo University, Ningbo, Zhejiang, China. ${ }^{4}$ Department of Tumor HIFU Therapy, HwaMei Hospital, University of Chinese Academy of Sciences, Ningbo, Zhejiang, China. ${ }^{5}$ Department of General Surgery, HwaMei Hospital, University of Chinese Academy of Sciences, Ningbo, Zhejiang, China. ${ }^{6}$ Department of Radiotherapy and Chemotherapy, Hwamei Hospital, University of Chinese Academy of Sciences, Northwest Street 41, Haishu District, Ningbo 315010 Zhejiang, China.
\end{abstract}

Received: 9 February 2021 Accepted: 4 June 2021

Published online: 21 June 2021

\section{References}

1. GBD 2017 Pancreatic Cancer Collaborators. The global, regional, and national burden of pancreatic cancer and its attributable risk factors in 195 countries and territories, 1990-2017: a systematic analysis for the Global Burden of Disease Study 2017. Lancet Gastroenterol Hepatol. 2019;4(12): 934-47.

2. Siegel RL, Miller KD, Jemal A. Cancer Statistics, 2017. CA Cancer J Clin. 2017; 67(1):7-30. https://doi.org/10.3322/caac.21387.

3. Vincent A, Herman J, Schulick R, Hruban RH, Goggins M. Pancreatic cancer. Lancet. 2011;378(9791):607-20.

4. Van Cutsem E, Aerts R, Haustermans K, Topal B, Van Steenbergen W, Verslype C. Systemic treatment of pancreatic cancer. Eur J Gastroenterol Hepatol. 2004;16(3):265-74. https://doi.org/10.1097/00042737-20040300000004.

5. Cartwright TH, Parisi M, Espirito JL, Wilson TW, Pelletier C, Patel M, et al. Clinical outcomes with first-Line chemotherapy in a large retrospective study of patients with metastatic pancreatic cancer treated in a US community oncology setting. Drugs Real World Outcomes. 2018;5(3):14959. https://doi.org/10.1007/s40801-018-0137-x.

6. Burris HA 3rd, Moore MJ, Andersen J, Green MR, Rothenberg ML, Modiano $M R$, et al. Improvements in survival and clinical benefit with gemcitabine as first-line therapy for patients with advanced pancreas cancer: a randomized trial. J Clin Oncol. 1997;15(6):2403-13. https://doi.org/10.1200/JCO.1997.15. 6.2403.

7. Halbrook CJ, Pontious C, Kovalenko I, Lapienyte L, Dreyer S, Lee HJ, et al, Macrophage-released pyrimidines inhibit gemcitabine therapy in pancreatic cancer. Cell Metab. 2019;29(6):1390-1399.e1396.

8. Ychou M, Conroy T, Seitz JF, Gourgou S, Hua A, Mery-Mignard D, et al. An open phase I study assessing the feasibility of the triple combination: oxaliplatin plus irinotecan plus leucovorin/5-fluorouracil every 2 weeks in patients with advanced solid tumors. Annals of oncology. 2003;14(3):481-9. https://doi.org/10.1093/annonc/mdg119.

9. Modulation of fluorouracil by leucovorin in patients with advanced colorectal cancer: evidence in terms of response rate. Advanced Colorectal Cancer Meta-Analysis Project. J Clin Oncol. 1992;10(6):896-903. https://doi. org/10.1200/JCO.1992.10.6.896.

10. Conroy T, Desseigne F, Ychou M, Bouché O, Guimbaud R, Bécouarn Y, et al. FOLFIRINOX versus gemcitabine for metastatic pancreatic cancer. N Engl J Med. 2011;364(19):1817-25. https://doi.org/10.1056/NEJMoa1011923.

11. Moher D, Shamseer L, Clarke M, Ghersi D, Liberati A, Petticrew M, et al. Preferred reporting items for systematic review and meta-analysis protocols (PRISMA-P) 2015 statement. Syst Rev. 2015;4(1):1. https://doi.org/10.1186/204 6-4053-4-1.

12. Gu L, Khadaroo PA, Chen L, Li X, Zhu H, Zhong X, et al. Comparison of long-term outcomes of endoscopic submucosal dissection and surgery for early gastric cancer: a systematic review and meta-analysis. J Gastrointest Surg. 2019:23(7):1493-501. https://doi.org/10.1007/s11605-019-04227-8.

13. Gu L, Yao Q, Shen Z, He Y, Ng DM, Yang T, et al. Comparison of tenofovir versus entecavir on reducing incidence of hepatocellular carcinoma in chronic hepatitis B patients: a systematic review and meta-analysis. J Gastroenterol Hepatol. 2020;35(9):1467-76. https://doi.org/10.1111/jgh.15036.

14. Du N, Chen M, Shen Z, Li S, Chen P, Khadaroo PA, et al. Comparison of quality of life and nutritional status of between Roux-en-Y and Billroth-I reconstruction after distal gastrectomy: a systematic review and metaAnalysis. Nutr Cancer. 2020;72(5):849-57. https://doi.org/10.1080/01635581.2 019.1656262.

15. Bigna JJ, Kenne AM, Asangbeh SL, Sibetcheu AT. Prevalence of chronic obstructive pulmonary disease in the global population with HIV: a systematic review and meta-analysis. Lancet Glob Health. 2018;6(2):e193202. https://doi.org/10.1016/S2214-109X(17)30451-5.

16. Fusar-Poli P, Cappucciati M, Borgwardt S, Woods SW, Addington J, Nelson B, et al. Heterogeneity of psychosis risk within individuals at clinical high risk: a meta-analytical stratification. JAMA Psychiatry. 2016;73(2):113-20. https://doi. org/10.1001/jamapsychiatry.2015.2324.

17. Gu L, Chen M, Guo D, Zhu H, Zhang W, Pan J, et al. PD-L1 and gastric cancer prognosis: a systematic review and meta-analysis. PloS one. 2017; 12(8):e0182692. https://doi.org/10.1371/journal.pone.0182692.

18. Yao Q, Gu L, Su R, Chen B, Cao H. Efficacy and safety of combination PD-1/ PD-L1 checkpoint inhibitors for malignant solid tumours: a systematic review. J Cell Mol Med. 2020;24(22):13494-506. https://doi.org/10.1111/ jcmm.15991.

19. Badiyan SN, Olsen JR, Lee AY, Yano M, Menias CO, Khwaja S, et al. Induction chemotherapy followed by concurrent full-dose gemcitabine and intensitymodulated radiation therapy for borderline resectable and locally advanced pancreatic adenocarcinoma. Am J Clin Oncol. 2016:39(1):1-7. https://doi. org/10.1097/COC.0000000000000003.

20. Javed MA, Beyer G, Le N, Vinci A, Wong H, Palmer D, et al. Impact of intensified chemotherapy in metastatic pancreatic ductal adenocarcinoma (PDAC) in clinical routine in Europe. Pancreatology. 2019;19(1):97-104. https://doi.org/10.1016/j.pan.2018.10.003.

21. Kang J, Hwang I, Yoo C, Kim KP, Jeong JH, Chang HM, et al. Nab-paclitaxel plus gemcitabine versus FOLFIRINOX as the first-line chemotherapy for patients with metastatic pancreatic cancer: retrospective analysis. Invest New Drugs. 2018;36(4):732-41. https://doi.org/10.1007/s10637-018-0598-5.

22. Kim S, Signorovitch JE, Yang H, Patterson-Lomba O, Xiang CQ, Ung B, et al. Comparative effectiveness of nab-paclitaxel plus gemcitabine vs FOLFIRINOX in metastatic pancreatic cancer: a retrospective nationwide chart review in the United States. Adv Ther. 2018;35(10):1564-77. https://doi. org/10.1007/s12325-018-0784-Z

23. Kordes M, Yu J, Malgerud O, Gustafsson Liljefors M, Löhr J. Survival Benefits of Chemotherapy for Patients with Advanced Pancreatic Cancer in A Clinical Real-World Cohort. Cancers (Basel). 2019;11(9):1326.

24. Lee JC, Woo SM, Shin DW, Kim J, Yang SY, Kim MJ, et al. Comparison of FOLFIRINOX and gemcitabine plus nab-paclitaxel for treatment of metastatic pancreatic cancer: using Korean Pancreatic Cancer (K-PaC) registry. Am J Clin Oncol. 2020:43(9):654-9. https://doi.org/10.1097/COC. 0000000000000730.

25. Muranaka T, Kuwatani M, Komatsu $Y$, Sawada K, Nakatsumi $H$, Kawamoto $Y$, et al. Comparison of efficacy and toxicity of FOLFIRINOX and gemcitabine with nab-paclitaxel in unresectable pancreatic cancer. J Gastrointest Oncol. 2017;8(3):566-71. https://doi.org/10.21037/jgo.2017.02.02. 
26. Orlandi A, Calegari MA, Martini M, Cocomazzi A, Bagalà C, Indellicati G, et al. Gemcitabine versus FOLFIRINOX in patients with advanced pancreatic adenocarcinoma hENT1-positive: everything was not too bad back when everything seemed worse. Clin Transl Oncol. 2016;18(10):988-95. https://doi. org/10.1007/s12094-015-1471-z.

27. Papneja N, Zaidi A, Chalchal H, Moser M, Tan K, Olson C, et al. Comparisons of outcomes of real-world patients with advanced pancreatic cancer treated with FOLFIRINOX versus gemcitabine and nab-paclitaxel: a populationbased cohort study. Pancreas. 2019;48(7):920-6. https://doi.org/10.1097/ MPA.0000000000001340.

28. Perri G, Prakash L, Qiao W, Varadhachary GR, Wolff R, Fogelman D, et al. Response and survival associated with first-line FOLFIRINOX vs gemcitabine and nabpaclitaxel chemotherapy for localized pancreatic ductal adenocarcinoma. JAMA Surg. 2020;155(9):832-9. https://doi.org/10.1001/jamasurg.2020.2286.

29. Rasmussen LS, Fristrup CW, Jensen BV, Pfeiffer P, Weber B, Yilmaz MK, et al. Initial treatment and survival in 4163 Danish patients with pancreatic cancer: a nationwide unselected real-world register study. Eur J Cancer. 2020;129:50-9

30. Tahara J, Shimizu K, Otsuka N, Akao J, Takayama Y, Tokushige K. Gemcitabine plus nab-paclitaxel vs. FOLFIRINOX for patients with advanced pancreatic cancer. Cancer Chemother Pharmacol. 2018;82(2):245-50. https:// doi.org/10.1007/s00280-018-3611-y.

31. Terashima $T$, Yamashita $T$, Sakai A, Ohta H, Hinoue $Y$, Toya D, et al. Treatment patterns and outcomes of unresectable pancreatic cancer patients in real-life practice: a region-wide analysis. Jpn J Clin Oncol. 2018; 48(11):966-73. https://doi.org/10.1093/jjco/hyy132.

32. Toesca DAS, Ahmed F, Kashyap M, Baclay JRM, von Eyben R, Pollom EL, et al. Intensified systemic therapy and stereotactic ablative radiotherapy dose for patients with unresectable pancreatic adenocarcinoma. Radiother Oncol. 2020;152:63-9. https://doi.org/10.1016/j.radonc.2020.07.053.

33. Wang Y, Camateros P, Cheung WY. A real-world comparison of FOLFIRINOX, gemcitabine plus nab-paclitaxel, and gemcitabine in advanced pancreatic cancers. J Gastrointest Cancer. 2019;50(1):62-8. https://doi.org/10.1007/s1202 9-017-0028-5.

34. Williet N, Saint A, Pointet AL, Tougeron D, Pernot S, Pozet A, et al. Folfirinox versus gemcitabine/nab-paclitaxel as first-line therapy in patients with metastatic pancreatic cancer: a comparative propensity score study. Ther Adv Gastroenterol. 2019;12:1756284819878660.

35. Suker M, Beumer BR, Sadot E, Marthey L, Faris JE, Mellon EA, et al. FOLFIRINOX for locally advanced pancreatic cancer: a systematic review and patient-level meta-analysis. Lancet Oncol. 2016;17(6):801-10. https://doi. org/10.1016/S1470-2045(16)00172-8.

36. Chen Z, Lv Y, Li H, Diao R, Zhou J, Yu T. Meta-analysis of FOLFIRINOX-based neoadjuvant therapy for locally advanced pancreatic cancer. Med. 2021; 100(3):e24068. https://doi.org/10.1097/MD.0000000000024068.

37. Kurata $Y$, Shiraki T, Ichinose $M$, Kubota K, Imai Y. Effect and limitation of neoadjuvant chemotherapy for pancreatic ductal adenocarcinoma: consideration from a new perspective. World J Surg Oncol. 2021;19(1):85. https://doi.org/10.1186/s12957-021-02192-8.

38. Mini E, Nobili S, Caciagli B, Landini I, Mazzei T. Cellular pharmacology of gemcitabine. Ann Oncol. 2006;17(Suppl 5).v7-12. https:/doi.org/10.1093/annond/mdj941.

39. Binenbaum Y, Náara S, Gil Z Gemcitabine resistance in pancreatic ductal adenocarcinoma. Drug Resist Updat 2015;23:55-68. https//doi.org/10.1016/j.drup.2015.10.002

40. Gandhi V, Legha J, Chen F, Hertel LW, Plunkett W. Excision of 2',2'difluorodeoxycytidine (gemcitabine) monophosphate residues from DNA. Cancer Res. 1996:56(19):4453-9.

41. Ceppi P, Volante M, Novello S, Rapa I, Danenberg KD, Danenberg PV, et al. ERCC1 and RRM1 gene expressions but not EGFR are predictive of shorter survival in advanced non-small-cell lung cancer treated with cisplatin and gemcitabine. Ann Oncol. 2006;17(12):1818-25. https://doi.org/10.1093/annonc/mdl300.

42. Akita H, Zheng Z, Takeda Y, Kim C, Kittaka N, Kobayashi S, et al. Significance of RRM1 and ERCC1 expression in resectable pancreatic adenocarcinoma. Oncogene. 2009;28(32):2903-9. https://doi.org/10.1038/onc.2009.158.

43. Giannatempo P, Pond GR, Sonpavde G, Raggi D, Naik G, Galsky MD, et al. The impact of adding taxanes to gemcitabine and platinum chemotherapy for the first-line therapy of advanced or metastatic urothelial cancer: a systematic review and meta-analysis. Eur Urol. 2016;69(4):624-33. https://doi. org/10.1016/j.eururo.2015.09.051.

44. Conroy T, Hammel P, Hebbar M, Ben Abdelghani M, Wei AC, Raoul JL, et al. FOLFIRINOX or gemcitabine as adjuvant therapy for pancreatic cancer. N Engl J Med. 2018;379(25):2395-406. https://doi.org/10.1056/NEJMoa1809775.
45. Ueno H, Okusaka T, Funakoshi A, Ishii H, Yamao K, Ishikawa O, et al. A phase II study of weekly irinotecan as first-line therapy for patients with metastatic pancreatic cancer. Cancer Chemother Pharmacol. 2007;59(4):447-54. https:// doi.org/10.1007/s00280-006-0283-9.

46. Wagener DJ, Verdonk HE, Dirix LY, Catimel G, Siegenthaler P, Buitenhuis M, et al. Phase II trial of CPT-11 in patients with advanced pancreatic cancer, an EORTC early clinical trials group study. Ann Oncol. 1995;6(2):129-32. https:// doi.org/10.1093/oxfordjournals.annonc.a059107.

47. Azrak RG, Cao S, Slocum HK, Tóth K, Durrani FA, Yin MB, et al. Therapeutic synergy between irinotecan and 5-fluorouracil against human tumor xenografts. Clin Cancer Res. 2004;10(3):1121-9. https://doi.org/10.1158/10780432.CCR-0913-3.

48. Mans DR, Grivicich I, Peters GJ, Schwartsmann G. Sequence-dependent growth inhibition and DNA damage formation by the irinotecan-5fluorouracil combination in human colon carcinoma cell lines. Eur J Cancer. 1999;35(13):1851-61.

49. Mullany S, Svingen PA, Kaufmann SH, Erlichman C. Effect of adding the topoisomerase I poison 7-ethyl-10-hydroxycamptothecin (SN-38) to 5fluorouracil and folinic acid in HCT-8 cells: elevated dTTP pools and enhanced cytotoxicity. Cancer Chemother Pharmacol. 1998;42(5):391-9. https://doi.org/10.1007/s002800050835.

50. Pavillard V, Formento P, Rostagno P, Formento $J$, Fischel JL, Francoual M, et al. Combination of irinotecan (CPT11) and 5-fluorouracil with an analysis of cellular determinants of drug activity. Biochem Pharmacol. 1998;56(10): 1315-22. https://doi.org/10.1016/S0006-2952(98)00205-6.

51. Ducreux M, Mitry E, Ould-Kaci M, Boige V, Seitz JF, Bugat R, et al. Randomized phase II study evaluating oxaliplatin alone, oxaliplatin combined with infusional 5-FU, and infusional 5-FU alone in advanced pancreatic carcinoma patients. Ann Oncol. 2004;15(3):467-73. https://doi. org/10.1093/annonc/mdh098.

52. Zeghari-Squalli N, Raymond E, Cvitkovic E, Goldwasser F. Cellular pharmacology of the combination of the DNA topoisomerase I inhibitor SN-38 and the diaminocyclohexane platinum derivative oxaliplatin. Clin Cancer Res. 1999;5(5):1189-96.

53. Conroy T, Paillot B, François E, Bugat R, Jacob JH, Stein U, et al. Irinotecan plus oxaliplatin and leucovorin-modulated fluorouracil in advanced pancreatic cancer--a Groupe Tumeurs Digestives of the Federation Nationale des Centres de Lutte Contre le Cancer study. J Clin Oncol. 2005; 23(6):1228-36. https://doi.org/10.1200/JCO.2005.06.050

54. Ciccolini J, Mercier C, Dahan L, André N. Integrating pharmacogenetics into gemcitabine dosing--time for a change? Nat Rev Clin Oncol. 2011;8(7):43944. https://doi.org/10.1038/nrclinonc.2011.1.

55. Jardim DL, Rodrigues CA, Novis YAS, Rocha VG, Hoff PM. Oxaliplatin-related thrombocytopenia. Ann Oncol. 2012;23(8):1937-42. https://doi.org/10.1093/a nnonc/mds074.

56. Curtis BR, Kaliszewski J, Marques MB, Saif MW, Nabelle L, Blank J, et al. Immunemediated thrombocytopenia resulting from sensitivity to oxaliplatin. Am J Hematol. 2006;81(3):193-8. https://doi.org/10.1002/ajh.20516.

57. Overman MJ, Maru DM, Charnsangavej C, Loyer EM, Wang H, Pathak P, et al. Oxaliplatin-mediated increase in spleen size as a biomarker for the development of hepatic sinusoidal injury. J Clin Oncol. 2010;28(15):2549-55. https://doi.org/10.1200/JCO.2009.27.5701.

58. Chikhladze S, Lederer AK, Kousoulas L, Reinmuth M, Sick O, Fichtner-Feigl S, et al. Adjuvant chemotherapy after surgery for pancreatic ductal adenocarcinoma: retrospective real-life data. World J Surg Oncol. 2019;17(1): 185. https://doi.org/10.1186/s12957-019-1732-3.

59. Kang H, Jo JH, Lee HS, Chung MJ, Bang S, Park SW, et al. Comparison of efficacy and safety between standard-dose and modified-dose FOLFIRINOX as a first-line treatment of pancreatic cancer. World J Gastrointest Oncol. 2018;10(11):421-30. https://doi.org/10.4251/wjgo.v10.i11.421.

60. Ghorani E, Wong HH, Hewitt C, Calder J, Corrie P, Basu B. Safety and efficacy of modified FOLFIRINOX for advanced pancreatic adenocarcinoma: a UK single-centre experience. Oncology. 2015;89(5):281-7. https:/doi.org/10.1159/000439171.

61. Feo CF, Deiana G, Ninniri C, Cherchi G, Crivelli P, Fancellu A, et al. Vascular resection for locally advanced pancreatic ductal adenocarcinoma: analysis of long-term outcomes from a single-centre series. World J Surg Oncol. 2021; 19(1):126. https://doi.org/10.1186/s12957-021-02238-x.

\section{Publisher's Note}

Springer Nature remains neutral with regard to jurisdictional claims in published maps and institutional affiliations. 